\title{
Immunity to candida infection: an overview
}

\begin{abstract}
Candida is found as a commensal of gastrointestinal and genital tract. However in contrast to many other commensals, Candida possesses a prominent ability to invade any tissue of a host presenting with innate or acquired immune deficiency. Candida spp. is an opportunistic pathogen that has acquainted various virulence traits facilitating host tissue colonization and invasion, and impairment of host defenses. The immune system is a remarkably versatile defense system that protects an individual from invasion of pathogens including Candida. Host defense mechanisms against candidiasis involve activation of an acute inflammatory response by innate immunity, followed by specific T cell (cell mediated immunity) or B cell (humoral immunity) mediated immune responses.
\end{abstract}

Keywords: candida, cell mediated immunity, defence mechanism, humoral immunity, immune response
Volume 5 Issue I - 2017

\author{
Sachin C Deorukhkar \\ Department of Microbiology, Rural Medical College, Pravara \\ Institute of Medical Sciences (Deemed University), India
}

Correspondence: Sachin C Deorukhkar, epartment of Microbiology, Rural Medical College, Pravara Institute of Medical Sciences (Deemed University), Loni, Maharashtra, India, , Tel 919850775564, Fax 91-2422-273442,

Email deorukhkar.sachin@gmail.com

Received: January 06, 2017| Published: January 20, 2017

\begin{abstract}
Abbreviations:
AMI,

antibody

mediated immunity; PMNL, polymorphonuclear leucocytes; CMI, cellmediated immunity; : chronic mucocutaneous candidiasis; GI, gastrointestinal; NK, natural killer; GM-CSF, granulocyte-macrophage colony stimulating factor; MPC, mononuclear phagocytic cells; PRPs, pattern recognition receptors; VVC, vulvovaginal candidiasis; TLRs, toll-like receptors; CLRs, C-type lectin receptors; RVVC, recurrent vulvovaginal candidiasis
\end{abstract}

\section{Introduction}

Infectious diseases have always been a feature of human life and continue to be a significant problem for health care professionals across the globe. Until the 19th century majority of infectious diseases were attributed to bacterial, parasitic and viral origins whereas role of fungi in human infections was less frequently recognized or documented. However the last three decades have witnessed a significant rise in the incidence of infections due to fungal pathogens. ${ }^{1}$

Among various opportunistic fungal infections, candidiasis has the greatest impact due to its frequency and the severity of complications associated with it. Candidiasisis the most opportunistic mycotic infection worldwide. Candida is found as a commensal of gastrointestinal and genital tract. However in contrast to many other commensals, Candida possesses a prominent ability to invade any tissue of a host presenting with innate or acquired immune deficiency. ${ }^{2}$

Candida spp. is the only mycotic pathogen capable of causing broad spectrum of clinical manifestations ranging from mucocutaneous overgrowth to disseminated infections. ${ }^{3}$ Although Candida can initiate infection in both immunocompetent and immunocompromised host, the incidence of candidiasis is usually high in immunocompromised individuals. Therefore, candidiasis can be rightly called as 'disease of diseased'. ${ }^{4}$

Candida spp. is an opportunistic pathogen that has acquainted various virulence traits facilitating host tissue colonization and invasion, and impairment of host defenses. ${ }^{5}$ The immune system is a remarkably versatile defense system that protects an individual from invasion of pathogens including Candida. The aim of this article is to comprehensively discuss the immune mechanisms involved in protection of host against Candida infection.

\section{Immunity to Candida infection}

The establishment of Candida infection in a susceptible host requires a series of well coordinated events to circumvent host's immunity. Host defense mechanisms against candidiasis involve activation of an acute inflammatory response by innate immunity, followed by specific $\mathrm{T}$ cell (cell mediated immunity) or B cell (humoral immunity) mediated immune responses. ${ }^{6}$

Although all arms of host's immune system are implicated in the control of candidiasis, predominance of type of immunity is highly dependent on the site and type of infection.?

Innate immunity by polymorphonuclear leucocytes (PMNL) and macrophages play a crucial role in protection against invasive Candida infections whereas; predominance of cell-mediated immunity (CMI) is noted in control of mucosal infections. ${ }^{7}$ The role of antibody mediated immunity (AMI) in candidiasis remains largely controversial. $^{7}$

Outcome of interaction between host's immune system and Candida spp. may either result in the elimination of infecting pathogen from the immunocompetent host, or development of persistent infection like chronic mucocutaneous candidiasis (CMC), candidemia and/or persistent systemic Candida infections in immunosuppressed host. ${ }^{6}$

\section{Innate immunity}

Physical and anatomic barriers that restrict the entry of pathogens inside the host tissue are considered as host's first line of defence against infection. The skin and the surface of mucus membranes are included in this category. Normal skin produces multiple substances like free fatty acids that prevent growth and multiplication of Candida spp. ${ }^{8}$

Variety of cytokines and chemokines inhibitory to Candida spp are secreted by epithelial cells ${ }^{7}$. Progessive shedding of surface epithelial cells prevents the adhesion of yeast cells. Endothelial cells can phagocytose Candida cells. ${ }^{7}$

Various mechanisms control the proliferation of Candida spp. within gastrointestinal (GI) tract. Salivary flow prevents the adherence of yeasts to the mucosal surfaces ${ }^{9}$. The flow and composition of saliva prevents Or opharyngeal candidiasis in healthy individuals by maintaining a dynamic equilibrium between Candida spp and other commensal flora. ${ }^{10}$

A variety of non-specific antimicrobial factors present in saliva contribute to innate immunity against Candida spp. These include lysozyme, lactoperoxidase histatins, calprotectin and lactoferrin. ${ }^{10}$ 
Lactoferrin acts as a chelating agent that competes with ora microorganisms for free ion radicals, which are essential for bacterial and fungal multiplication. In addition, it also damages the fungal cell wall and activates intracellular autolytic enzymes. ${ }^{10}$

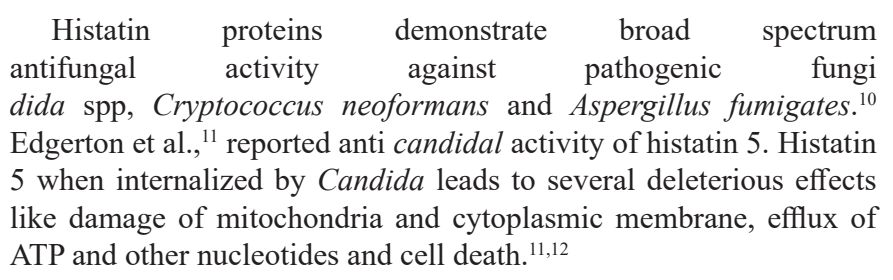

Calprotectin is heterodimeric calcium and zinc binding protein produced by PMNL, monocytes, macrophages and mucosal keratinocytes. It ceases the Candida growth by depriving the fungus of zinc. ${ }^{10}$ Investigation of Steinbakk et al. (1990) demonstrated in vitro inhibitory action of calprotectin on growth of Candida spp..$^{10}$

Commensal bacterial flora of GI tract inhibit Candida proliferation by various mechanisms like competition for nutritional and ecological niche or site of adherence, unfavourable alternation in environmental conditions and production of toxic by-products. ${ }^{8}$

Of various mechanisms responsible for control of Candida by colonising flora, competition for nutrition is considered as the most important one. ${ }^{13}$ Depletion of commensal bacterial flora by broad spectrum antibiotic therapy is one of the well established risk factors for candidiasis. ${ }^{8,13}$

Once the Candida spp. penetrate the GI mucosa and get access to the host tissue, a variety of serum factors are activated. The surface proteins of Candida strongly stimulate all 3 (classical, alternative and mannose binding lectin (MBL)) complement cascade activation. ${ }^{14}$ Complement activation leads to opsonization and intracellular killing of Candida spp.

The alternative complement pathway, activated by cell wall components of Candida spp is primarily responsible for enhanced phagocytosis of yeast cells. ${ }^{8}$ MBL cascade plays an important role in opsonization, phagocytosis and other complement functions. Both $\mathrm{C} 3 \mathrm{~b}$ and $\mathrm{C} 3 \mathrm{~d}$ fragments are capable of binding C. albicans. ${ }^{8}$

The interaction between activated $\mathrm{C} 3 \mathrm{~b}$ and the complement receptor CR3 facilitates phagocytosis of Candida cells. C5 also play an important role in immunity against Candida infections. Activation of $\mathrm{C} 5$ triggers the formation of $\mathrm{C} 5 \mathrm{~b}$, which facilitates the phagocytosis and release of terminal complement components. ${ }^{14}$ Binding of Candida surface proteins like Gpm1, Pra 1 and Gpd2 prevents recognition and phagocytosis..$^{15}$ Deficiency of complement leads to poor host resistance against candidiasis. Activation of complement also regulates series of signaling events during disseminated infections. ${ }^{14}$

PMNL are considered to be the most important host defence mechanism against invasive candidiasis. ${ }^{7}$ This is evidenced by the high incidence of systemic Candida infections like candidemia in patients with prolonged neutropenia or neutrophil disorders. ${ }^{16}$ Neutrophils are the only immunocyte that prevent the transition of Candida spp. from yeast to filamentous form. ${ }^{14}$ This type of leucocyte also controls the elimination of Candida spp. from the blood stream. PMNL additionally express various pattern recognition receptors as well as receptors for opsonizing antibodies and complement components. Among various mechanisms responsible for PMN mediated killing of Candida spp., the 'oxidative burst' appears to be the most prominent. Oxidative burst is the process of rapid formation of reactive oxygen intermediates. ${ }^{14,15}$ This process requires assembly of the NADPH oxidase enzyme complex in the cytoplasmic or phagosomal membrane to release superoxide. ${ }^{14,15}$

Natural killer (NK) cell system is another important innate host defence mechanism against candidiasis. The candidacidal activity of NK cells is largely due to production of granulocyte-macrophage colony stimulating factor (GM-CSF). NK cells also produce factors responsible for activation of mononuclear phagocytic cells (MPC) and PMNL. These immune cells have only marginal cytotoxic effect on C. albicans.

The ability of PMNL and MPC to phagocytose the Candida cell, doen't means that they necessarily destroy the yeast. Although phagocytes are capable of killing most yeast cells and small hyphae, some yeast however escape this process and proliferate within the phagocytes. Mechanisms like inhibition of production of reactive oxygen species, prevention of phagolysosome fusion and increased $\mathrm{pH}$ are responsible for protection of yeast from phagocytosis. ${ }^{16}$ Additionally, transformation of yeast into the filamentous form in the cytoplasm of immune cells, inhibits mitosis and leads to apotosis. ${ }^{16}$

\section{Cell-mediated immunity}

CMI appears play an important role in prevention of mucosal candidiasis as patients with T-cell deficiencies (e.g. HIV/AIDS patients) are at high risk of developing of mucosal candidiasis, but rarely develop disseminated candidiasis. ${ }^{17}$ High incidence of Candida mucosal infections is seen in HIV infected patients, transplant recipients and individuals on corticosteroid therapy. ${ }^{7}$

A strong correlation is noted between OPC and reduced blood CD4+ cell counts. In HIV infected individuals depletion of CD4+ cells below a critical threshold of 200 cells $/ \mathrm{mm} 3$ most often triggers the onset of OPC. ${ }^{10}$ Mucocutaneous overgrowth of Candida spp. leading to infections can also occur in patients with idiopathic CD4 lymphocytopenia or after anti-CD52 monoclonal antibody therapy. ${ }^{13}$

Type $1 \mathrm{~T}$ helper (Th1) responses confers protection against candidiasis, whereas Th2 type responses lack the ability to inhibit fungal growth and multiplication or may also increase susceptibility to infections. Therefore a shift from Th1 to Th2 response in the peripheral circulation may enhance the risk of OPC. ${ }^{7}$

Candida cell wall components like mannan and $\beta-1,3-\mathrm{D}$-glucan are pathogen-associated molecular patterns (PAMPs) that are recognized by pattern recognition receptors (PRRs) present on surface of phagocytes. ${ }^{6}$ PRRs are grouped into several families like the Tolllike receptors (TLRs), the C-type lectin receptors (CLRs) and the nucleotide-binding domain leucine-rich repeat-containing receptors (NLRs). ${ }^{6}$

Interaction between PAMPs and PRRs induce differentiation of Th into Th 17 phenotype..$^{13}$ Cytokines (IL-17 and IL-22) secreted by Th 17 , trigger the production of antimicrobial peptides known as $\beta$-defensins. $\beta$-defensins controls the overgrowth of colonizing Candida. The release of IL-17 and IL-22 also recruits and activates neutrophils. These activated neutrophils lead to elimination of Candida infection. ${ }^{6}$ Inability to produce IL-17 and IL-22 predisposes patients suffering from rare immunodeficiency diseases like hyper IgE/Job's syndrome and chronic mucocutaneous candidiasis to mucocutaneous candidiasis. ${ }^{13}$ Studies of various researchers have documented protective role of IL17 in mucocutaneous and disseminated candidiasis. IL-17 is known to reduce fungal load. ${ }^{15}$

In contrast to $\mathrm{OPC}$, the role of $\mathrm{CMI}$ in vulvovaginal candidiasis (VVC) and recurrent vulvovaginal candidiasis is less significant. The prevalence of VVC in HIV infected women is not more common 
compared to HIV non-infected. Patients suffering from recurrent vulvovaginal candidiasis (RVVC) rarely develop OPC or other forms of mucocutaneous Candida infections similarly; patients with CMC or OPC do not suffer from VVC or RVVC. ${ }^{7}$ IL-22 plays a vital role in restricting the growth of fungus and maintaining epithelial barrier function. The polymorphisms in the IL-22 gene associated with protection against RVVC can be correlated with elevated IL-22 level and decreased levels of IL-17 and TNF $\alpha .{ }^{15}$

Recently, Navarro-Arias et al., ${ }^{18}$ reported that mannans are relevant for the C. guilliermondii-host interaction. Disruption of Protein mannosylation affects $C$. guilliermondii Cell Wall, Immune Sensing, and Virulence. ${ }^{18}$

\section{Humoral immunity}

Although different antibodies classes like IgG, IgM or IgA are produced (except in highly immunocompromised patients) in all clinical types of candidiasis, the protective role of humoral immunity is largely unknown. In fact, patients with mucosal candidiasis (HIV non- infected with RVVC and HIV infected with OPC) demonstrate normal or elevated levels of anti-Candida antibodies. ${ }^{7}$

Till date, there are no studies, showing direct evidence of increased susceptibility to mucocutaneous and systemic candidiasis in patients with pure B-cell abnormalities (either congenital or acquired).

$\operatorname{IgG}$ and $\operatorname{IgM}$ are found in sera of patients with mucocutaneous and deep- seated candidiasis. Additionally, these antibodies can be also seen in during asymptomatic colonization in low titers. Few earlier studies have reported rise in salivary $\operatorname{IgA}$ and $\operatorname{IgG}$ antibodies in patients with candidiasis. Anti-mannan and anti-glucan antibodies are demonstrated in sera. Their protective role is however uncertain because elevated levels of these antibodies are seen in a poor prognosis of infections. ${ }^{2}$

Coogan et al. (1994) noted rise in salivary antibodies in patients with HIV/AIDS compared with controls..$^{10}$ They suggested that in an HIV infected individual the antibody titer appears to reflect responses to Candida rather than protection. These salivary immunoglobulins are believed to interfere with the adherence of Candida to the salivary mucosa. However, this mechanism collapses with the increase in the load of yeast cells.

Secretory $\operatorname{Ig} \mathrm{A}(\mathrm{s} \operatorname{Ig} \mathrm{A})$ represent as the predominant immunoglobulin isotype in saliva and other secretion and is considered as most important immunoglobulin type for defense of pathogens that colonize or invade surfaces bathed by external surfaces. However, the protective role of SIgA remains non compatible with rarity of OPC in patients with selective IgA deficiency. ${ }^{10}$

\section{Conclusion}

The establishment of Candida infection in a susceptible host requires a series of well coordinated events to circumvent host's immunity. Host defence mechanisms against candidiasis involve activation of an acute inflammatory response by innate immunity, followed by specific $\mathrm{T}$ cell (cell mediated immunity) or B cell (humoral immunity) mediated immune responses. Outcome of interaction between host's immune system and Candida spp. may either result in the elimination of infecting pathogen from the immunocompetent host, or development of persistent infection like chronic mucocutaneous candidiasis (CMC), candidemia and/or persistent systemic Candida infections in immunosuppressed host.

\section{Acknowledgments}

None.

\section{Conflicts of interest}

Author declares there are no conflicts of interest.

\section{Funding}

None.

\section{References}

1. Rayner MG, Zhang Y, Gorry MC, et al. Evidence of Bacterial metabolic activity in culture- Negative otitis media with effusion. JAMA. 1998;279(4):296-290

2. Srinivasan A, Lopez-Ribot JL, Ramasubramanian AK. Overcoming antifungal resistance. Drug Discov Today Technol. 2014;11:65-71.

3. Poulain D, Jouault T. Candida albicans cell wall glycans, host receptors and responses: elements for decisive crosstalk. 2004;7(4):342-349.

4. Deorukhkar SC, Saini S, Mathew S. Virulence factors contributing to pathogenicity of Candida tropicalis and its antifungal susceptibility profile. International Journal of Microbiology. 2014;2014(2014):6.

5. Deorukhkar SC, Saini S. Candidiasis: Past, present and future. Int J Infect Trop Dis. 2015;2:12-24.

6. Deorukhkar SC, Saini S, Mathew. Non-albicans Candida Infection: An emerging threat. Interdisciplinary Perspectives on Infectious Diseases. 2014;2014(2014):7.

7. Netea MG, Marodi L. Innate immune mechanisms for recognition and uptake of Candida species. Trends Immunol. 2010;31(9):346-353.

8. Fidel Jr PL. Immunity to Candida. Oral Dis . 2002;8(suppl 2):69-75.

9. Greenfield RA. Host defense system interaction with Candida. J Med Vet Mycol. 1992;30(2):89-104.

10. Enwonwu CO, Meeks VI. Oral candidiasis, HIV, and saliva glucocorticoids. Am J Pathol. 1996;148(4):1313-1338.

11. de Repentigny L, Lewandowski D, Jolicoeur P. Immunopathogenesis of oropharyngeal candidiasis in human immunodeficiency virus infection. Clin Microbiol Rev. 2004;17(4):729-759.

12. Edgerton M, Koshlukova SE, Lo TE, et al. Candidacidal activity of salivary histatins. Identification of a histatin 5-binding protein on Candida albicans. J Biol Chem . 1998;273(32):20438-20447.

13. Helmerhorst EJ, van't Hof W, Breeuwer P, et al. Characterization of histatin 5 with respect to amphipathicity, hydrophobicity, and effects on cell and mitochondrial membrane integrity excludes a candidacidal mechanism of pore formation. J Biol Chem. 2001;276(8):5643-5649.

14. van der Meer JW, van de Veerdonk FL, Joosten LA, et al. Severe Candida spp. infections: new insights into natural immunity. Int J Antimicrob Agents. 2010;36(Suppl 2):S58-S62.

15. Duggan S, Leonhardt I, Hünniger K, et al. Host response to Candida albicans bloodstream infection and sepsis. Virulence. 2015;6 (4):316-326.

16. Naglik JR. Candida Immunity. New J Sci . 2014;2014(2014):27.

17. Demirezen Ş, Dönmez HG, Özcan M,et al. Evaluation of the relationship between fungal infection, neutrophil leukocytes and macrophages in cervicovaginal smears: Light microscopic examination. J Cytol . 2015;32(2):79-84.

18. Ha JF, Italiano $\mathrm{CM}$, Heath $\mathrm{CH}$, et al. Candidemia and invasive candidiasis: A review of the literature for the burns surgeon. Burns. 2011;37(2):181-195.

19. Navarro-Arias MJ, Defosse TA, Dementhone K, et al. Disruption of Protein Mannosylation Affects Candida guilliermondii Cell Wall, Immune Sensing, and Virulence. Front Microbiol . 2016;7:1951. 